http://jmscr.igmpublication.org/home/ ISSN (e)-2347-176x ISSN (p) 2455-0450 crossref DOI: https://dx.doi.org/10.18535/jmscr/v8i4.44

\title{
A Drug Utilization Study of Antimicrobials in Major Surgical Patients in Tertiary Care Teaching Hospital- A Prospective Observational Study
}

\author{
Authors \\ Dr Vidisha Vivek Parulekar ${ }^{*}$, Dr Vandana Avinash Badar², \\ Dr Vartika Gupta ${ }^{3}$, Dr Priti Garate ${ }^{4}$
}

${ }^{1,3,4}$ Resident, Department of Pharmacology, Indira Gandhi Government Medical College, Nagpur

${ }^{2}$ Associate Professor, Department of Pharmacology, Indira Gandhi Government Medical College, Nagpur

*Corresponding Author

Dr Vidisha Vivek Parulekar

Resident, Department of Pharmacology, Indira Gandhi Government Medical College, Nagpur, India

\begin{abstract}
Introduction: Surgical site infection (SSI) is the most common post-operative complication and represents a significant burden in terms of patient morbidity and mortality, and cost to health services around the world. Appropriate antibiotic prophylaxis has been shown to be effective in reducing the incidence of surgical site infections as studies suggest that 40-60\% of these infections are preventable.

Aims: To evaluate and compare the utilization pattern of antimicrobial agents (AMA) in surgical prophylaxis use in preoperative, intraoperative and post-operative wards of different specialities.

Settings and Design: It is a cross-sectional observational study conducted patients admitted to surgery, orthopaedics, ENT and gynaecology wards in tertiary teaching care hospital.

Methods and Material: The study duration was from November 2016-November 2018 (2 years).The data was collected time to time from all the medical records of the patient. The study protocol was approved by the Institutional Ethics Committee.

Statistical Analysis Used: Data was collected and compiled using Microsoft Excel 2010 and then analysed by calculating simple proportions, frequency and percentage of various parameters.

Results: Out of 386 patients, it was observed that female patients (55.69\%) were more than male (44.60\%) patients. The majority of surgical wounds were clean (39.11\%) and most common antimicrobial used pre-operatively was metronidazole (29.24\%) and in the intraoperative and postoperative period was gentamicin (54.28\%). Number of DDD for inj.amoxicillin-clavulanic acid $1.2 \mathrm{~g}$ was 274.4 .

Conclusions: After observing the trend of use of antibiotics, there is a clear need for the development of prescribing guidelines and educational initiatives to encourage the appropriate use of antimicrobials in surgical period. Also, there is scope for improving prescribing habits among the fraternity and minimizing incidence of resistance to antimicrobials.

Keywords: surgical site infection, pre-operative, intra-operative, post-operative, antibiotic prophylaxis.
\end{abstract}

\section{Introduction}

Surgical site infection (SSI) is the most common post-operative complication and represents a significant burden in terms of patient morbidity and mortality, and cost to health services around the world. They are the second commonest 
nosocomial infection accounting for approximately one quarter of 2 million hospital acquired infections in USA annually ${ }^{1}$. Between $1.9 \%$ and $2.7 \%$ of all surgical patients, more than 500,000 per year, are diagnosed with SSI leading to an estimate of 8000 annual deaths , $^{2,3,4,5}$.

Antibiotic consumption in humans is increasing globally. The greatest increase between 2000 and 2010 was in low- and middle-income countries, but in general, high-income countries still use more antibiotics per capita. Growing economic prosperity and rising incomes, as well as expanding insurance coverage, have increased antibiotic consumption. Inappropriate use of broad spectrum antimicrobials leads to decrease in sensitivity of antimicrobials against microorganisms. With increasing multidrug resistance and limited availability of newer agents, there is urgent need for vigilant surveillance, stringent infection control practices, as well as rational antibiotic prescription.

In India, there are National Treatment guidelines for Antimicrobial use in infectious diseases given by National Centre for disease Control (NCDC), Directorate General of Health Services, Ministry of Health \& Family Welfare, Government of India $(2016)^{6}$ and Treatment Guidelines for Antimicrobial Use in Common Syndromes given by Indian Council of Medical Research (ICMR), Department of Health Research, New Delhi, $2017^{7}$.

Antibiotic prophylaxis can protect the patients from post-operative infections by abating the bacterial load present within the surgical site at the time of operation ${ }^{8}$. Hence, prophylactic antibiotics should be administered before giving an incision to any intra-operative patient.

Appropriate antibiotic prophylaxis has been shown to be effective in reducing the incidence of surgical site infections as studies suggest that 40$60 \%$ of these infections are preventable ${ }^{9}$. Also it results in good hospital functioning, which leads to increase in patient's satisfaction, maintains high reputation for the hospital, fewer hospitalizations, lesser incidence of re-surgeries and re-admissions and less cost for care and treatment, as well as lesser numbers of claims and compensations ${ }^{1}$. Also there are very few studies which describe the utilization of drugs postoperatively.

Therefore, the present study was conducted to analyse the rationality and drug utilization patterns of antimicrobials in perioperative surgical inpatients and to compare with the standard treatment guidelines (National treatment guidelines for antimicrobials) and also calculate the defined daily dosing (DDD) of antimicrobials as per WHO ATC-DDD criteria.

\section{Subjects and Methods}

The study was prospective observational conducted in indoor patients of various surgical departments in Tertiary care teaching institute in central India from November 2016-November 2018 (2 years). The study did not involve any interview of the patients admitted. The study protocol was approved by the Institutional Ethics Committee prior to the commencement of the study. The inclusion criteria was patients above 18years, of either gender, patient undergoing major surgeries, patient receiving in-patient care in departments of surgery, gynecology, orthopedics and ENT, patients having comorbidities like diabetes, hypertension, malnutrition, COPD, TB, HIV, obesity, smoking and alcohol abuse. The exclusion criteria was pregnancy and lactation, medico-legal cases, patient receiving in-patient care in cardiothoracic, ophthalmic, neurosurgical, pediatric surgical, obstetrics departments, any patient who dies postoperatively before being discharged, patient who absconded/discharged against medical advice, patient referred to other hospitals, incomplete data. The data from 386 surgical in-patients was collected from their prescriptions \& peri-operative notes admitted in departments of general surgery (4 units, 5 wards- 3 male ward and 2 female ward and 1 surgical intensive care unit [SICU]), gynecology (3 units and 5 wards), orthopedics (3 units and 2 wards-one male and one female ward) and ENT ( 2 units and 2 wards- one male and one 
female ward). The in-charge authority of surgical departments was notified and permission was taken.

Demographic analysis of data was done. Data was analysed according to classification of wound by CDC (Centers for Disease Control and Prevention) into clean, clean-contaminated, contaminated and dirty surgical wounds ${ }^{10}$ and as per type of surgery into abdominal, gynecology, head and neck, musculoskeletal etc.

Following parameters like WHO prescribing indicators ${ }^{11}$, most common AMA used in clean, clean-contaminated, contaminated and dirty surgical wounds, percentage of AMAs used as generic drugs, branded drugs, fixed dose combinations (FDCs) and DCGI (Drug Controller General of India) approved FDCs and route of administration of AMAs used peri-operatively were also included.

To measure the degree to which practices adhere to rational therapy as well as standard treatment guidelines, percentage of AMA prescribed from an essential medicine list (EML) or National list of essential medicine (NLEM) was calculated with following formula.

Percentage calculated $(\%)=\underline{\text { No. of products prescribed which are in essential drug list } \mathrm{x}} 100$

Total number of drugs prescribed

From the prescription data of commonly prescribed groups of drugs, the amounts of drugs consumed were converted into the number of
DDD as per the 2010 version of ATC/DDD index $^{12}$.

\section{No of DDD $=\underline{\text { No. of items issued } \times \text { Amount of the drug per item }}$ \\ WHO recommended DDD of drug}

Comparison with standard treatment guidelines such as National treatment guidelines for of antimicrobial use in infectious diseases by $\mathrm{NCDC}^{6}$ and Treatment Guidelines for Antimicrobial Use in Common Syndromes by $\mathrm{ICMR}^{7}$ were also included.

\section{Statistical Analysis}

Data was collected and compiled using Microsoft Excel 2010 and then analysed by calculating simple proportions, frequency and percentage of various parameters. Suitable graphs, charts and photographs were added. A brief interpretation was included in the results below every table. Sample Size was calculated by using software Open Epi, Version 3, an open source calculator and was 386 considering population size $(\mathrm{N})$ that is the total number of major operations done in surgical OT, gynecology OT, ENT OT and orthopedics OT for last one year i.e. from February 2016 to February 2017 as 3971 and prevalence as $50 \%$.

\section{Results}

In our study, 386 patients were followed up till the postoperative period. It was observed that female to male ratio of 1.21 as shown in Figure 1. The age-wise distribution of data was categorized in Figure 2. 
Figure 1 Gender-wise distribution of data

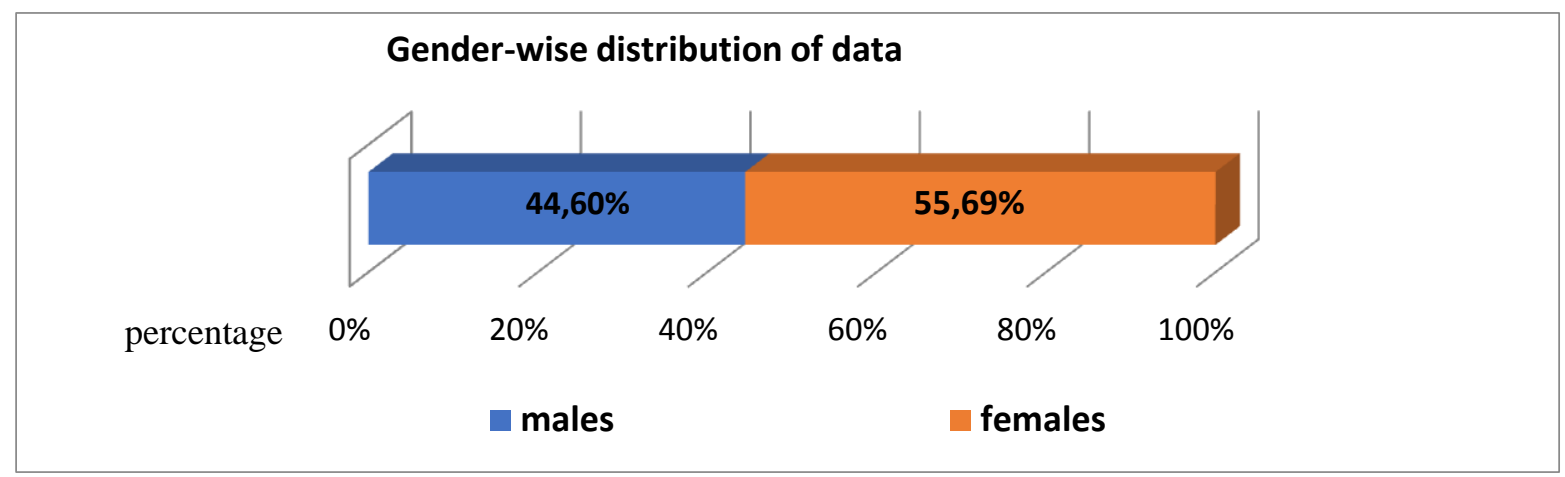

Figure 2 Age-wise distribution of data

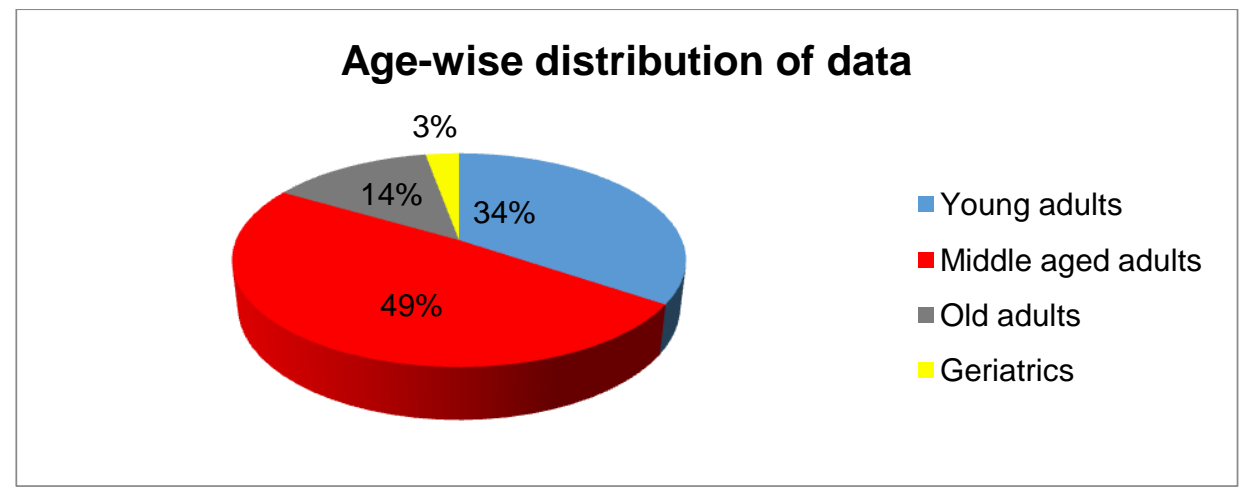

In figure 3, department-wise distribution of data is shown. Four departments were involved in the study with majority of cases from general surgery department (43\%).

Figure 3 Department-wise distribution of data

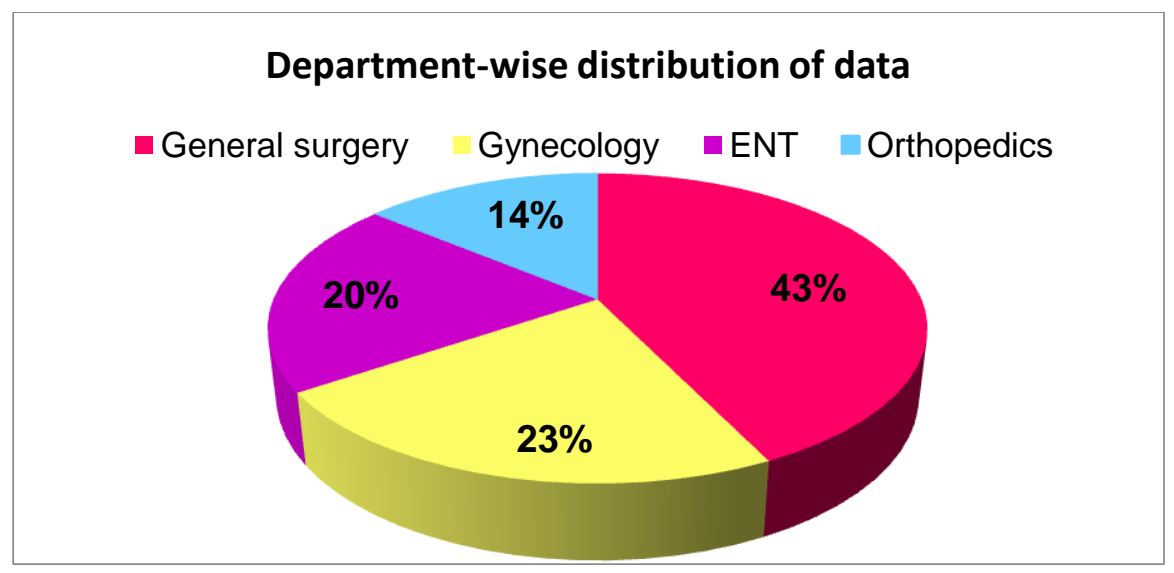

The data was analysed according to the type of surgical wound as shown in Table 1.

Table 1 Classification of surgical wound

\begin{tabular}{|l|c|c|}
\hline \multicolumn{3}{|l|}{ Classification of surgical wound $(\mathrm{n}=386)$} \\
\hline Type of surgical wound & No of patients & Percentage of patients (\%) \\
\hline 1- clean & 151 & 39.11 \\
\hline 2- clean-contaminated & 120 & 31.08 \\
\hline 3- contaminated & 75 & 19.43 \\
\hline 4- dirty & 40 & 10.36 \\
\hline
\end{tabular}

The common surgeries associated with clean surgical wound were operations of head and neck surgeries like tonsillectomy, modified radical mastoidectomy and musculoskeletal surgeries like 
fracture fixations or arthroplasties, hernia repair surgeries. Clean-contaminated surgical wounds included operations like gynaecological operative procedures such as myomectomies, hysteroscopies, hysterectomies etc; urological surgeries such as lithotripsy, pylotithotomy, nephrectomy. Contaminated surgical wounds comprised of rectal surgeries like haemorrhoidectomies, fistulectomies and abdominal surgeries. Dirty surgical wounds included exploratory laparotomy for intraabdominal abscess or perforation peritonitis and scrotal surgeries like chylocoele or pyocoele.

As shown in Table 2; majority of study cases were of abdominal surgery with $24.61 \%$, followed by gynecology surgery with $22.79 \%$ and head and neck surgeries with $20.46 \%$.

Table 2 Type of surgery

\begin{tabular}{|c|c|c|}
\hline \multicolumn{3}{|l|}{ Type of surgery $(n=386)$} \\
\hline Type of surgery & No of patients & Percentage of patients (\%) \\
\hline Abdominal surgery & 95 & 24.61 \\
\hline Gynecology surgery & 88 & 22.79 \\
\hline Head and neck surgery & 79 & 20.46 \\
\hline Musculoskeletal surgery & 54 & 13.98 \\
\hline Rectal surgery & 26 & 6.73 \\
\hline Hernia repair surgery & 18 & 4.66 \\
\hline Scrotal surgery & 12 & 3.10 \\
\hline Urological surgery & 10 & 2.59 \\
\hline Breast surgery & 4 & 1.03 \\
\hline
\end{tabular}

On evaluation of WHO prescribing parameters it was seen that the average number of AMA

prescribed per prescription was 1.8 as shown in Table 3.

Table 3 WHO prescribing parameters

\begin{tabular}{|l|c|}
\hline Total drugs prescribed & 3059 \\
\hline $\begin{array}{l}\text { Percentage of AMA used by generic } \\
\text { name/prescription }\end{array}$ & $79.56 \%$ \\
\hline Average number of drugs/prescription & 4.854922 \\
\hline Average number of AMA/prescription & 1.8 \\
\hline $\begin{array}{l}\text { Percentage of AMA prescribed for } \\
\text { Intravenous route }\end{array}$ & $81.09 \%$ \\
\hline $\begin{array}{l}\text { Percentage of AMA prescribed from } \\
\text { Essential medicine list (EML) }\end{array}$ & $97.14 \%$ \\
\hline $\begin{array}{l}\text { Percentage of AMA prescribed from National } \\
\text { list of essential medicine (NLEM) }\end{array}$ & $90.64 \%$ \\
\hline Average duration of SICU stay & 5.26 \\
\hline Average duration of hospital stay & 11.36 \\
\hline AMA - Antimicrobials
\end{tabular}

The total antimicrobials prescribed at preoperative, intra-operative and post-operative stage were 660, 35 and 844 respectively. Most common AMA used pre-operatively, intraoperatively and postoperatively was metronidazole (29.24\%), gentamicin $(54.28 \%)$ and metronidazole $(26.52 \%)$ respectively. Apart from amoxicillin-clavulanic acid, other fixed dose combinations seen were Piperacillin +Tazobactum 2.25g and 4.5g, Ampicillin $1 \mathrm{~g}+$ Sulbactam $0.5 \mathrm{~g}$ as shown in Table 4. 
Table 4 AMA prescribed during peri-operative period

\begin{tabular}{|c|c|c|}
\hline \multicolumn{3}{|l|}{ AMA prescribed at preoperative stage $(n=660)$} \\
\hline AMA & $\begin{array}{c}\text { No of antibiotics } \\
\text { prescribed }\end{array}$ & $\begin{array}{c}\text { Percentage of antibiotics } \\
\text { prescribed }(\%)\end{array}$ \\
\hline *Cap.amoxicillin-clavulanic acid $625 \mathrm{mg}$ & 38 & 5.75 \\
\hline inj.amikacin1g & 17 & 2.57 \\
\hline inj.amikacin500mg & 14 & 2.12 \\
\hline *inj.Augmentin (amoxicillin/clavulanic acid) $1.2 \mathrm{~g}$ & 104 & 15.75 \\
\hline *inj.ampicillin $1 \mathrm{~g}+$ sulbactam $0.5 \mathrm{~g}$ & 4 & 0.60 \\
\hline inj.cefotaxime $1 \mathrm{~g}$ & 47 & 7.12 \\
\hline inj.ceftriaxone $1 \mathrm{~g}$ & 137 & 20.75 \\
\hline inj.ciprofloxacin $200 \mathrm{mg}$ & 14 & 2.12 \\
\hline inj.gentamicin $80 \mathrm{mg}$ & 38 & 5.75 \\
\hline inj.levofloxacin 500mg & 4 & 0.60 \\
\hline inj.meropenem $1 \mathrm{~g}$ & 22 & 3.33 \\
\hline inj.metronidazole $100 \mathrm{mg}$ & 24 & 3.63 \\
\hline inj.metronidazole $250 \mathrm{mg}$ & 18 & 2.72 \\
\hline inj.metronidazole $500 \mathrm{mg}$ & 147 & 22.27 \\
\hline inj.ofloxacin $200 \mathrm{mg}$ & 4 & 0.60 \\
\hline *inj.piptaz (piperacillin/tazobactam) $4.5 \mathrm{~g}$ & 4 & 0.60 \\
\hline moxifloxacin drops $0.5 \%$ & 8 & 1.21 \\
\hline T.cefixime $200 \mathrm{mg}$ & 4 & 0.60 \\
\hline T.metronidazole $500 \mathrm{mg}$ & 4 & 0.60 \\
\hline T.albendazole 400mg & 4 & 0.60 \\
\hline T.moxifloxacin $400 \mathrm{mg}$ & 4 & 0.60 \\
\hline \multicolumn{3}{|l|}{ AMA prescribed at intra-operative stage $(n=35)$} \\
\hline inj.cefotaxime $1 \mathrm{~g}$ & 8 & 22.85 \\
\hline inj.ceftriaxone $1 \mathrm{~g}$ & 8 & 22.85 \\
\hline inj.gentamicin $80 \mathrm{mg}$ & 19 & 54.28 \\
\hline \multicolumn{3}{|l|}{ AMA prescribed at postperative stage $(n=841)$} \\
\hline *Cap.amoxicillin-clavulanic acid $625 \mathrm{mg}$ & 39 & 4.6 \\
\hline inj.amikacin 500mg & 19 & 2.25 \\
\hline inj.amikacin1g & 11 & 1.32 \\
\hline *inj.Augmentin (amoxicillin/clavulanic acid) $1.2 \mathrm{~g}$ & 138 & 16.56 \\
\hline *inj.ampicillin $1 \mathrm{~g}+$ sulbactam $0.5 \mathrm{~g}$ & 4 & 0.48 \\
\hline inj.cefotaxime $1 \mathrm{~g}$ & 54 & 6.48 \\
\hline inj.ceftriaxone $1 \mathrm{~g}$ & 157 & 18.84 \\
\hline inj.ciprofloxacin 100mg & 20 & 2.4 \\
\hline inj.ciprofloxacin 500mg & 3 & 0.36 \\
\hline inj.ciprofloxacin $200 \mathrm{mg}$ & 8 & 0.96 \\
\hline inj.gentamicin $80 \mathrm{mg}$ & 57 & 6.84 \\
\hline inj.levofloxacin $750 \mathrm{mg}$ & 3 & 0.36 \\
\hline inj.levofloxacin 500mg & 8 & 0.96 \\
\hline inj.meropenem $1 \mathrm{~g}$ & 25 & 3 \\
\hline inj.metronidazole $100 \mathrm{mg}$ & 40 & 4.8 \\
\hline inj.metronidazole $250 \mathrm{mg}$ & 20 & 2.4 \\
\hline inj.metronidazole 500mg & 161 & 19.32 \\
\hline inj.ofloxacin $200 \mathrm{mg}$ & 4 & 0.48 \\
\hline *inj.piptaz (piperacillin/tazobactam) $4.5 \mathrm{~g}$ & 34 & 4.04 \\
\hline inj. teicoplanin $400 \mathrm{mg}$ & 8 & 0.96 \\
\hline moxifloxacin drops $0.5 \%$ & 8 & 0.96 \\
\hline T.ciprofloxacin $500 \mathrm{mg}$ & 8 & 0.96 \\
\hline T.doxycycline $100 \mathrm{mg}$ & 4 & 0.48 \\
\hline T.moxifloxacin $400 \mathrm{mg}$ & 4 & 0.48 \\
\hline
\end{tabular}


Figure 4 AMA used in clean surgical wounds

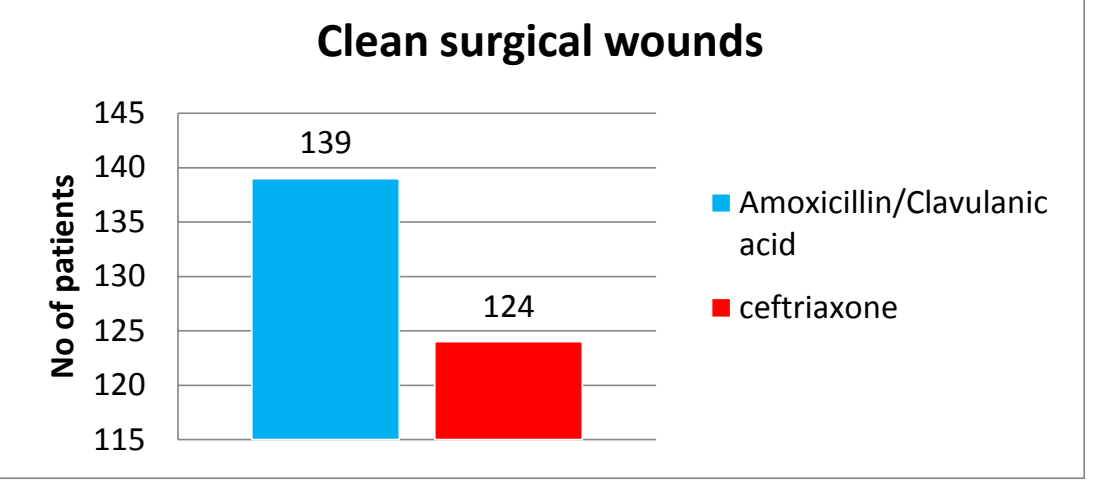

Figure 5 AMA used in clean contaminated surgical wounds

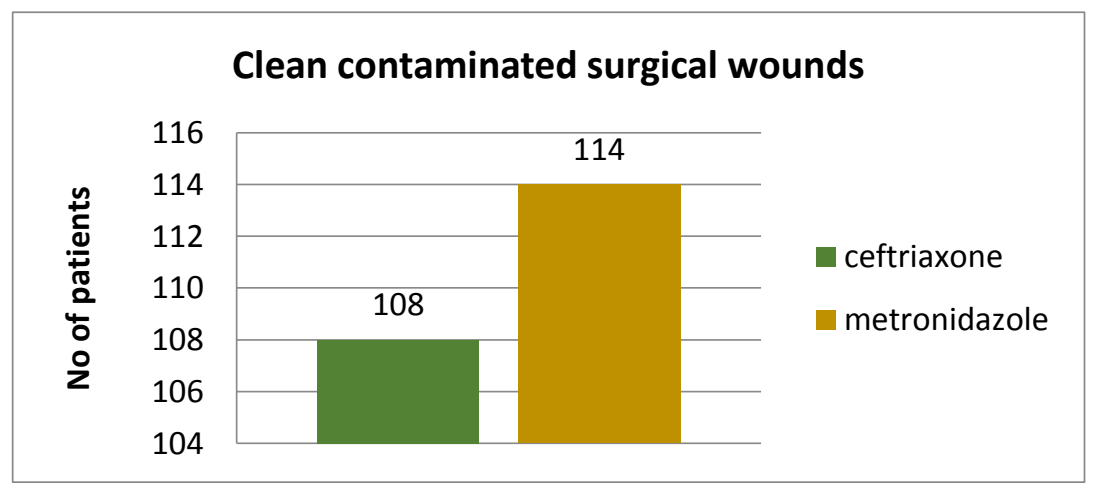

Figure 6 AMA used in contaminated surgical wound

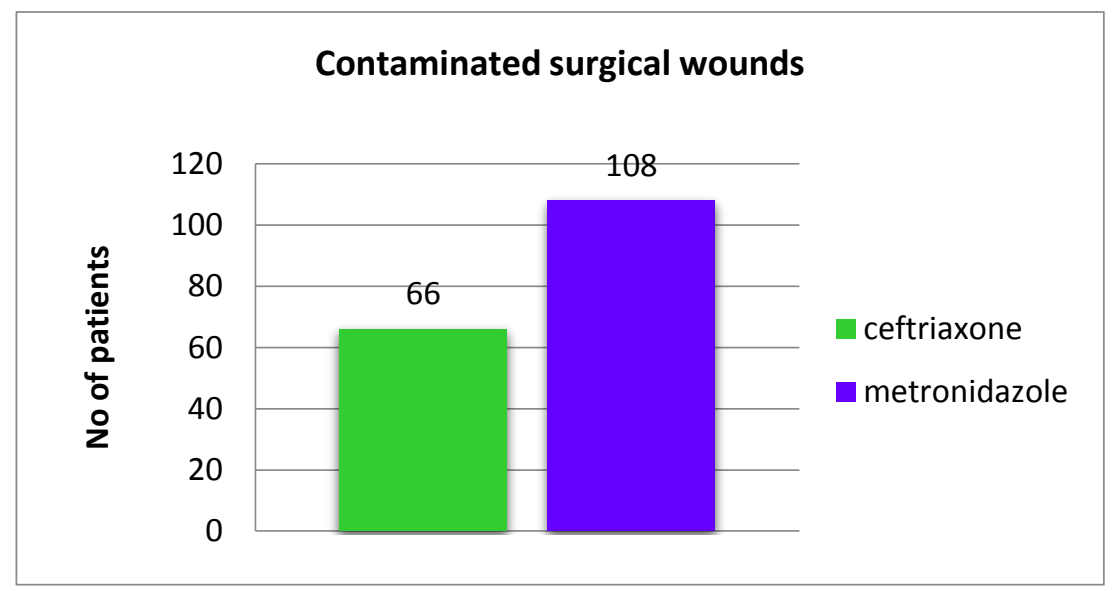

Figure 7 AMA used in dirty surgical wounds

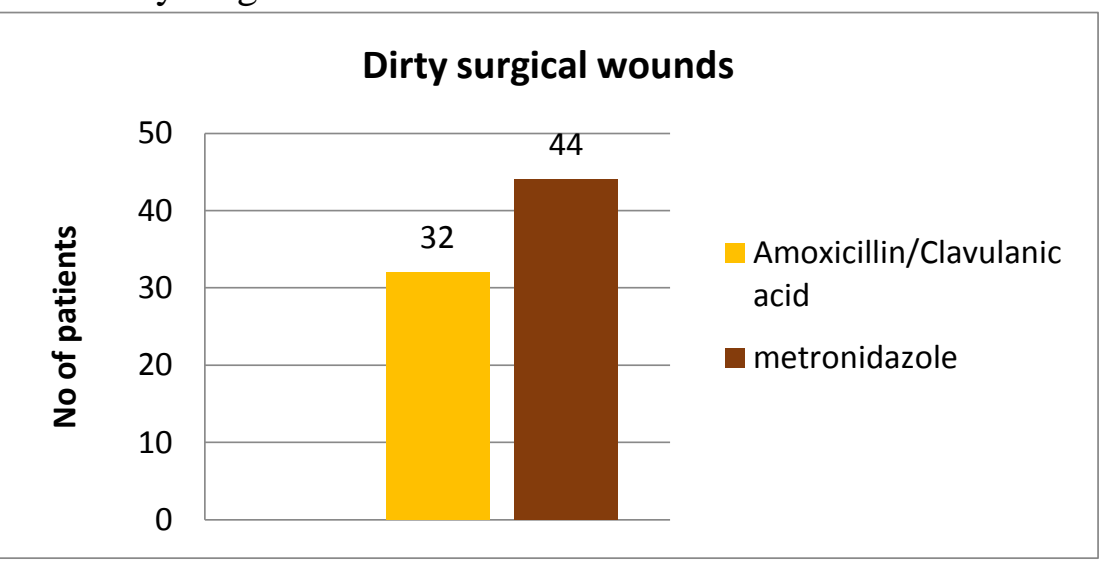


Maximum number of drugs were prescribed by fixed dose combinations as shown in table 5. generic name $(79.89 \%)$. $13.76 \%$ of drugs were

Table 5 Use of generic drugs, branded drugs \& fixed dose combinations

\begin{tabular}{|l|c|c|}
\hline \multicolumn{3}{|c|}{ Use of generic drugs, branded drugs \& fixed dose combinations (n=3059) } \\
\hline Drugs & Number & Percentage (\%) \\
\hline Generic drugs & 2444 & 79.89 \\
\hline Branded drugs & 615 & 20.10 \\
\hline Fixed dose combinations & 421 & 13.76 \\
\hline FDC approved by DCGI & 338 & 80.28 \\
\hline FDC approved by WHO & 318 & 75.53 \\
\hline
\end{tabular}

FDC- Fixed dose combinations, DCGI- Drug Controller General of India.

Maximum study medications were prescribed by intravenous route in peri-operative period $(81.03 \%)$ as shown in Figure 8.

Figure 8 Routes of administration of AMAs used peri-operatively

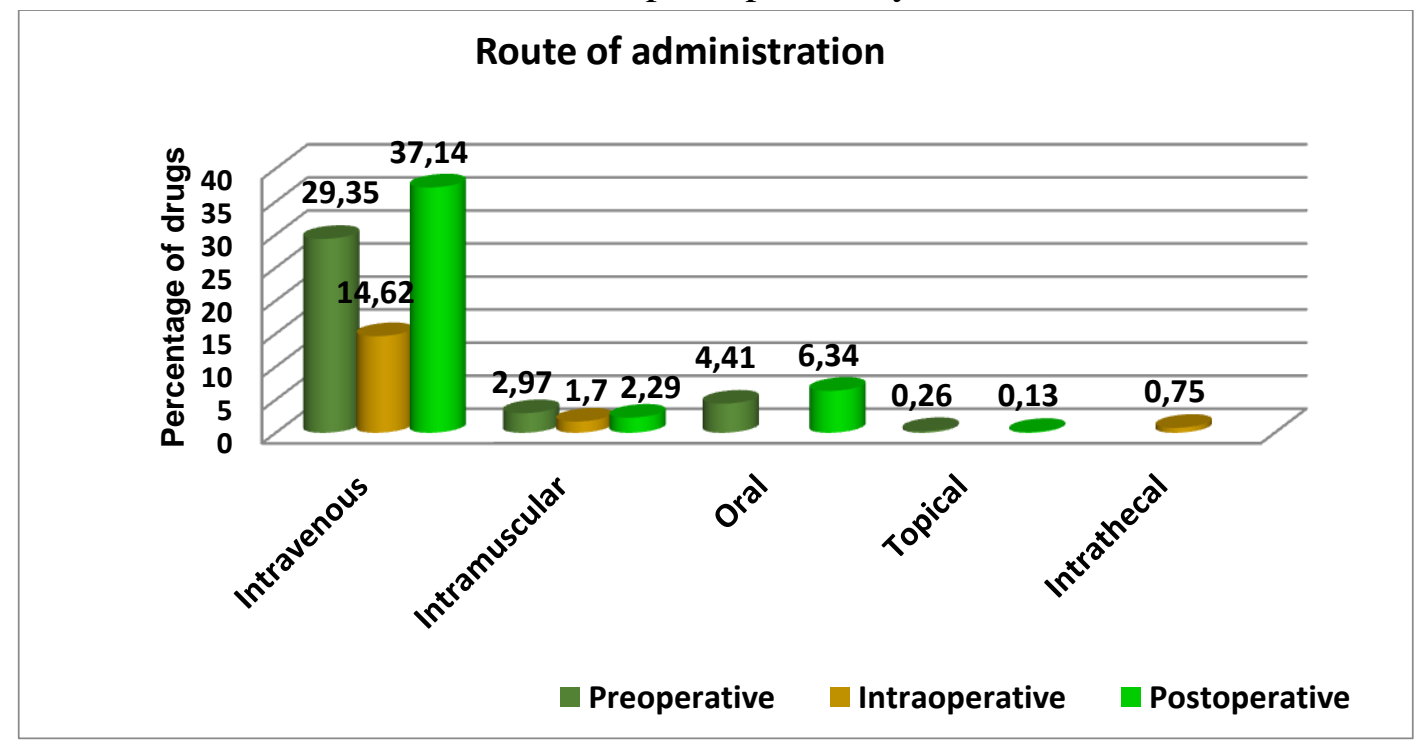

For the common 3 AMAs used in the perioperative period, ATC code has been mentioned and number of DDD calculated as shown in Table 6.

Table 6 -ATC-DDD classification

\begin{tabular}{|l|c|c|c|}
\hline DRUG & ATC CODE & WHO recommended DDD & No. of DDD \\
\hline $\begin{array}{l}\text { Inj amoxicillin with clavulanic } \\
\text { acid(1.2g) }\end{array}$ & J01CR02 & $3 \mathrm{~g}$ & 274.4 \\
\hline Inj ceftriaxone(1g) & J01DD04 & $2 \mathrm{~g}$ & 528 \\
\hline Inj.metronidazole $(500 \mathrm{mg})$ & J01XD01 & $1.5 \mathrm{~g}$ & 325.3 \\
\hline
\end{tabular}

\section{Discussion}

In this study, the patients were admitted for various diagnosis like acute appendicitis, acute cholecystitis, acute tonsillitis, uterine myomas, dysfunctional uterine bleeding, fractures, osteoarthritis etc. According to demographic data, male to female ratio is 0.82 as oppose to Akter $S F$ et al where it was $1.81^{13}$. This is due to inclusion of cases operated in department of gynecology. The average age was 41.84 years and the most common age group was middle aged adults (3655years) with $49 \%$. This finding of our study is in consistent with study by Sharma and Goel where the mean age was $45.33 \pm 19.01$ years ${ }^{14}$. Similar results were also seen in the Bhataia et $a l^{15}$ and Khan $M$ L et al ${ }^{16}$ study. This is the usual trend as this is the productive age group and is actively involved in socioeconomic activities, which may lead to stress and age factor making them 
vulnerable to diseases that may need surgical interventions ${ }^{15}$.

Maximum number of cases were taken from wards of general surgery department (43\%) followed by gynaecology (14\%) department. This finding was similar to study by Alemkere $G$ were participants from general surgery were $60.1 \%$ followed by gynaecology cases with $24.8 \%{ }^{17}$. Also, $S G$ kamath et al noted a same finding with $32.47 \%$ of cases from general surgery department ${ }^{18}$.However in study by Rehan HS et al, most patients were admitted under General surgery department (30\%) followed by orthopedics department $(26 \%)^{19}$.

As per the CDC classification of surgical wounds, it was observed in our study that clean surgeries were the dominating ones with $39.11 \%$. Similar finding was noted in the study by Sozen $H$ et al $(\mathrm{n}=340)$ on perioperative antibiotic prophylaxis. They noted that clean surgeries were $31.8 \%{ }^{20}$. This might be because majority of cases were of hernia repair surgeries (hernioplasty), modified radical mastoidectomies, open reduction and internal fixations of fractures with nail or plate or pins or screws, total hip and knee arthroplasties, hemiarthroplasties, tonsillectomies and modified radical mastectomies which belonged to clean surgeries.

In our study, most common surgeries were abdominal surgeries with $24.61 \%$. This finding was not consistent with Patel DJ et al where they found out that commonest surgeries were urological procedures $(30.5 \%)$ and abdominal surgeries were only $18.5 \%(37 \text { cases })^{21}$. Many cases of abdominal surgeries like exploratory laparotomies, laparoscopic appendectomies, laparoscopic cholecystectomies were observed in our study.

Majority of AMA were prescribed by generic name $(79.56 \%)$. This finding is consistent with Bhataia et al where 5627 total medicine formulations were studied and $98.51 \%$ (5534) were found out to be prescribed by official/generic names ${ }^{15}$. Percentage of drugs prescribed by generic name according to the WHO standard should be $100 \%$. Increasing generic prescribing would rationalize the use and reduce the cost of drugs. It also reduces confusion while prescribing $^{22}$. Average number of AMA/ prescription in our study was 1.8. All patients received an antimicrobial drug at one time or the other. This is similar to Bhansali et $a^{23}$ and Kumar $R$ et $a l^{24}$ study But this is much higher than the other Indian studies which reports the use of AMA in $20 \%$ to $67 \%$ of the patients only ${ }^{15,23}$. Similar studies abroad indicate a range of $20 \%$ to $42 \%$ of patients receiving antimicrobial drugs ${ }^{25}$. The higher number of AMA per patient indicates that more and more antimicrobial were used for prophylaxis purpose rather than definitive treatment purpose and that they are used blanket therapy to prevent any or all types of infection. The percentage of AMAs prescribed from National list of essential medicine (NLEM) were $90.64 \%$. This finding was consistent with essential drug list of our hospital.

Overall the most common AMAs used perioperatively was metronidazole in our study. Also the most common antimicrobial used preoperatively in our study was metronidazole followed by ceftriaxone with and amoxicillinclavulanic acid. Similarly in Agrawal et al study, metronidazole topped the list ${ }^{26}$. Metronidazole is used predominantly to cover up for all the anaerobic infections ${ }^{25}$. In our study, all patients were given preoperative prophylactic antimicrobial which was similar to study done in Kerala $^{27}$ and Ahemadabad ${ }^{28}$.

Though in our study third generation cephalosporins were most commonly used for surgical prophylaxis, recent guidelines recommend the use of first generation cephalosporins as surgical antibiotic prophylaxis $^{29,30}$. First-generation cephalosporins particularly cefazolin are the most suitable agents for surgical prophylaxis due to their spectrum that includes Staphylococcus species and gramnegative bacilli along with desirable pharmacokinetic characteristics, ease of administration and low cost. But local resistance 
pattern, surgeon's own experience at hospital setting and availability of AMAs in our own government medical store might influence the choice of anti-microbial.

In our study amoxicillin and clavulanic acid found to have used in $44.7 \%, 54.4 \%$ and $50 \%$ of clean, clean contaminated and dirty surgical wounds respectively. The high rate of infection with amoxicillin and clavulanic acid in our study demonstrates need for updating the $\beta$-lactam inhibitor combinations as prophylaxis in surgery $^{31}$. But in Chandrasekaran et al study cefuroxime was used in $68.42 \%$ of clean surgical wounds, cefaperazone/sulbactum was most frequently used as $27.08 \%$ in clean-contaminated and $26.77 \%$ of contaminated cases as single dose administration of first and second generation cephalosporin is sufficient for optimal prevention of surgical site infections in absence of high rate of resistance ${ }^{31}$. Dirty wounds and Clean- contaminated wounds have more chances of getting infected as compared to clean wounds and contaminated ones have the highest rate of infection $^{29}$. Hence it was seen that more number of AMAs were used in dirty and clean contaminated wounds.

Very few prescriptions were based on laboratory culture sensitivity reports. According to ICMR guidelines, in culture confirmed cases of SSI, antimicrobials should be based on Lab AST reports ${ }^{7}$. Also prescription of antibiotics without evidence of culture and sensitivity tests is another major problem that results in poor patient compliance. Strict antibiotic prescription policies have to be developed and target must be aimed to minimize the incidence of resistance to antimicrobial agents and also to promote infection control practices and rational antibiotic utilization $^{32}$.

$80.28 \%$ and $75.53 \%$ of FDCs prescribed in our study were approved by DCGI and WHO respectively. This indicates the rational prescribing pattern.

In table 6, ATC-DDD classification of 3 most common AMAs. The DDD is the assumed average maintenance dose per day for a drug used for its main indication in adults. The DDD does not necessarily reflect the recommended or actual dose used ${ }^{33}$. The objective of ATC/DDD system is to serve as tool for drug utilization research, to favor improvement in drug use ${ }^{34}$.

The AMA use in our study does not align with the national guidelines for surgical prophylaxis and as the preference in the use of antimicrobials was due to availability of free drugs from hospital supply and hospital standard operating procedures. Selection of antimicrobial agent is done taking into consideration various aspects like site of surgery, spectrum and pharmacokinetic profile. No universal surgical antimicrobial prophylaxis guideline can be implemented basis solely on the type of surgical wound as the occurrence of SSIs is also influenced by other factors such as site and length of the procedure, overall health of the patient, i.e., glucose levels, weight of the patients, etc $^{30}$. The guidelines for prophylaxis are based on the evidence obtained from controlled clinical trials. These guidelines encourage the utilization of older narrow spectrum antimicrobials ${ }^{35}$.

\section{Limitations of the study}

The limitations of the current study include the involvement of small number of patients which did not give complete overview among the different departments. Further studies involving different departments encompassing superspeciality and oncology surgical patients should be established.

\section{Conclusion}

The present study illustrates the pattern and type of antimicrobial use in surgical departments of tertiary care hospital. It also highlights the use of drugs by generic name, use of drugs from the NLEM and EML with metronidazole being the most commonly used antimicrobial. There is lack of alignment in drug use when we compare with national guidelines. After observing the trend of use of antimicrobials, there is a clear need for the development of prescribing guidelines and 
educational initiatives to encourage the appropriate use of antimicrobials and analgesics in surgical period. Also, there is scope for improving prescribing habits among the fraternity and minimizing incidence of resistance to antimicrobials. A periodic survey by hospital infection control committee is essential. Awareness regarding antimicrobial resistance, timing and duration of surgical antimicrobial prophylaxis, optimal post-operative pain management should be imparted to all the prescribers.

\section{Author's contribution}

All authors have contributed equally in developing the concept of the study, data collection, data analysis, and drafting the manuscript.

\section{Acknowledgement}

Special thanks to Dr. Manish Nandeshwar sir, Dr. Neha Moghe and Dr. Pragati Sharma from department of pharmacology and microbiology of IGGMC, Nagpur respectively. The authors would like to be grateful for all the patients involved in this study.

\section{Conflict of interest}

Declared none.

\section{References}

${ }^{1}$ Stone PW. Economic burden of healthcareassociated infections: an American perspective. Expert review of pharmacoeconomics \& outcomes research. 2009 Oct 1;9(5):417-22.

${ }^{2}$ Meeks DW, Lally KP, Carrick MM, Lew DF, Thomas EJ, Doyle PD, Kao LS. Compliance with guidelines to prevent surgical site infections: as simple as 1-2-3?. The American Journal of Surgery. 2011 Jan 1;201(1):76-83.

${ }^{3}$ CDC. Data from National Hospital Discharge Survey. 2010. Available at : https://www.cdc.gov/nchs/data/nhds/4procedures/ 2010pro_numberpercentage.pdf.

Accessed

September 2018.

${ }^{4} \mathrm{Mu}$ Y, Edwards JR, Horan TC, Berrios-Torres SI, Fridkin SK. Improving risk-adjusted measures of surgical site infection for the National Healthcare Safely Network. Infection Control \& Hospital Epidemiology. 2011 Oct;32(10):970-86.

${ }^{5}$ Klevens RM, Edwards JR, Richards Jr CL, Horan TC, Gaynes RP, Pollock DA, Cardo DM. Estimating health care-associated infections and deaths in US hospitals, 2002. Public health reports. 2007 Mar;122(2):160-6.

${ }^{6}$ National Treatment Guidelines for Antimicrobial Use in Infectious Diseases (2016) http://pbhealth.gov.in/AMR_guideline700149588 9.pdf

${ }^{7}$ Treatment Guidelines for Antimicrobial Use in Common Syndromes [Internet]. New Delhi; 2017 [cited 28 November 2018]. Available from: https://www.icmr.nic.in/sites/default/files/guidelin es/treatment_guidelines_for_antimicrobial.pdf ${ }^{8}$ Bratzler DW, Hunt DR. The surgical infection prevention and surgical care improvement projects: national initiatives to improve outcomes for patients having surgery. Clinical infectious diseases. 2006 Aug 1;43(3):322-30.

${ }^{9}$ Hawn MT, Vick CC, Richman J, Holman W, Deierhoi RJ, Graham LA, Henderson WG, Itani KM. Surgical site infection prevention: time to move beyond the surgical care improvement program. Annals of surgery. 2011 Sep 1;254(3):494-501.

${ }^{10}$ Mangram AJ, Horan TC, Pearson ML, Silver LC, Jarvis WR, Hospital Infection Control Practices Advisory Committee. Guideline for prevention of surgical site infection, 1999. Infection Control \& Hospital Epidemiology. 1999 Apr;20(4):247-80.

${ }^{11}$ WHO. How to investigate drug use in health facilities: selected drug indicators, action program on essential drugs (DAP), Geneva, 1993. Available at: http://apps.who.int/medicinedocs/en/d/Js2289e/. Accessed on 19 September 2018. 
${ }^{12}$ WHOCC - Purpose of the ATC/DDD system [Internet]. Available from: https://www.whocc.no/atc_ddd_methodology/pur pose_of_the_atc_ddd_system/

${ }^{13}$ Akter SF, Rani MF, Rahman JA, Nordin MS, Satwi S, Awang MB. Antimicrobial use and factors influencing prescribing in medical wards of a tertiary care hospital in Malaysia. Int J Sci Environ Technol. 2012;1(4):274-84.

${ }^{14}$ Sharma P, Goel D. Utilization assessment of antimicrobial prophylaxis in surgical patients at tertiary care teaching hospital. Saudi Journal for Health Sciences. 2018 Jan 1;7(1):23.

${ }^{15}$ Bhataia S, Mathur S, Sankhla S, Kumar M, Sharma M. International Journal of Pharma and Bio Sciences ISSN 2016 April; 7(2):332 - 338.

${ }^{16}$ Khan MLZ, Arain A, Qureshi FA. Patient's selection in limited resources a stimulus for improving care. PJS2008, 24, (4), 35-8.

${ }^{17}$ Alemkere G. Antibiotic usage in surgical prophylaxis: A prospective observational study in the surgical ward of Nekemte referral hospital. PloS one. 2018 Sep 13;13(9):e0203523.

${ }^{18}$ Kamath SG, Varun HV, Rani DU, Aithal S and Patil UN: Prescribing patterns of antimicrobials in surgical departments in a Tertiary Care Hospital in South India. Int J Pharm Sci Res 2014; 5(3): 1051-58

${ }^{19}$ Rehan HS, Kakkar AK, Goel S. Pattern of surgical antibiotic prophylaxis in a tertiary care teaching hospital in India. International journal of infection control. 2010;6(2).

${ }^{20}$ Sozen H, Cetinkaya M, Sahan MU, Caylak SD, Hazer DB, Canbek UM, Koca YS, Baldemir E. Cost and efficacy of perioperative antibiotic prophylaxis in a state hospital in Turkey. Acta Medica. 2013 Jan 1;29:455-60.

${ }^{21}$ Patel DJ, Chhaiya SB, Mehta DS. Drug utilization study-pattern of use of anti-microbial drugs among post-operative patients in department of general surgery at a tertiary care hospital. Int J Basic Clin Pharmacol 2017;6:1482-5.

${ }^{22}$ Goodman L, Gilman A, Brunton L, HilalDandan R, Knollmann B, Buxton I. Principles of
Prescription Order Writing and Patient Compliance : Goodman \& Gilman's the pharmacological basis of therapeutics. 13th ed. New York [i 11 pozostałych]: McGraw-Hill Education; 2018.

${ }^{23}$ Bhansali NB, Gosai TR, Dholaria NK, Suthar SD, Chacko J,Chavda DA, et al. Drug utilization study in post-operative patients in surgical ward of a tertiary hospital attached with medical college. Der Pharm Lett. 2013; 5(1):251-7.

${ }^{24}$ Kumar R, Kohli Kamlesh, Sidhu D. S., Kaur Navjot, Mala Chandra, Garg Monique. An indepth study of drugs prescribing pattern in the Surgery Department of a Tertiary Care Teaching Institute in Northern India. Int J Basic ClinPharmacol. 2014 Aug; 3(4):681-686.

${ }^{25}$ Bhabhor P, Hotchandani H. An Antibacterial Drug Utilization Study at Surgical Units of Shree Sayaji General Hospital, Vadodara, Gujarat, India. The Internet J Pharmacol. 2012;10(1):1-11.

${ }^{26}$ Agrawal JM, Patel NM, Vaniya HV, Trivedi HR, Balat JD. Drug utilization study in postoperative patients in obstetrics and gynaecology ward of a tertiary care teaching hospital. J Clin Exp Res. 2014;2:103-109.

${ }^{27}$ Tourmousoglou CE, Yiannakopoulou EC, Kalapothaki V, Bramis J, St. Papadopoulos J. Adherence to guidelines for antibiotic prophylaxis in general surgery: A critical appraisal. J Antimicrob Chemother 2008;61:214- 8 .

${ }^{28}$ Khan AK, Mirshad PV, Rashed MR, Banu G. A study on the usage pattern of antimicrobial agents for the prevention of surgical site infections (SSIs) in a tertiary care teaching hospital. J Clin Diagn Res 2013;7:671- 4.

${ }^{29}$ Scotish Intercollegiate Guidelines Network. Updated April 2014 Antibiotic Prophylaxis in Surgery - A National Clinical Guideline; 2008. Available from: http://www.sign.ac.uk/pdf/sign104. [Last accessed on 2018 Nov 5].

${ }^{30}$ World Health Organization. Guidelines for Safe Surgery: Safe Surgery Saves Lives. Geneva: World Health Organization; 2009. Available from: 
http://www.who.int/patientsafety/safesurgery/tool

s_ resources/9789241598552/en/. [Last accessed on 2018 Nov 5].

${ }^{31}$ Chandrasekaran K, Saeed K, Gandhiraj D, Mohanta GP, Rajasekaran A. Study on Adherence to Prophylactic Antimicrobials Use Guidelines in Surgical Wards of an Indian Private Corporate Hospital. International Journal of Pharmaceutical Research and Allied Sciences. 2016 Jan 1;5(2):280-92.

${ }^{32}$ Divyashree L, Bhushan A and Tejaswini K. Drug utilisation study in gynaecological postoperative cases: a retrospective study. EJPMR.2017;4(04):418-423.

${ }^{33}$ Rode $\mathrm{S}$ and Salankar H: Analysis of antimicrobial usage pattern: a retrospective observational study. Int J Pharm Sci \& Res 2018; 9(3): 1231-36. doi: 10.13040/IJPSR.09758232.9(3).1231-36.

${ }^{34}$ Ali H, Zafar F, Alam S, Beg AE, Bushra R, Manzoor A, Naqvi GR, Yasmeen R, Shafiq Y, Tariq A, Zubair S. Drug utilization and prescribing pattern of antibiotics in a tertiary care setups; trends and practices. Pakistan journal of pharmaceutical sciences. 2018 Mar 2;31.

${ }^{35}$ R. Revathi, N. Saralaand A. Bhaskaran. Prescribing Pattern of Antimicrobials in Patients during Post-Operative Period - An Observational Study.British Journal of Medicine \& Medical Research. 2015;5(10):1213-1219. 